\title{
Computations of geometrically linear and nonlinear Ginzburg-Landau models for martensitic pattern formation
}

\author{
B.K. Muite ${ }^{1, a}$ and O.U. Salman ${ }^{2, b}$ \\ 1 Mathematical Institute, 24-29 St. Giles, University of Oxford, Oxford, OX1 3LB, UK \\ ${ }^{2}$ Laboratoire D'Etude des Microstructures, ONERA, 29 Avenue de la Division Leclerc, 92322 Chatil- \\ lon, France
}

\begin{abstract}
Computations show that a two dimensional geometrically nonlinear Ginzburg-Landau model with inertia exhibits long lived metastable states, that have martensite domains with split tips and bent needles similar to those observed in NiAl. In comparison, the geometrically linear model quickly relaxes to states with twins which extend all the way across the sample and have only short lived tip splitting and needle bending.
\end{abstract}

\section{Introduction}

Martensitic phase transformations involve a change of shape in the underlying crystal lattice from a high symmetry phase to several lower symmetry phases. These lead to complex microstructural patterns of twinned laminates that involve a wide range of length scales. These phase transformations can have strains greater than $10 \%$ and large rotations relative to the original undeformed state. Many dynamic simulations of models for martensitic phase transformations have used geometrically linear models under the assumption that geometric nonlinearities have a small effect $[1,11,21]$. Analytical studies of static energy minimizing geometrically nonlinear models and their geometrically linear counterparts indicate that there can be significant differences between the two models $[9,14,15]$. These studies do not show whether these differences are significant in physically relevant situations. For a review of frame indifference and the differences between geometrically linear and nonlinear elasticity see $[3,9,19,20,25]$.

\section{Viscoelastic models for martensitic phase transformations}

The square to rectangle martensitic transformation can be modeled using an isothermal energy comprised of kinetic, strain and interfacial terms,

$$
\int_{\Omega} \frac{\rho}{2} U_{t}^{2}+\Phi(\boldsymbol{F})+\frac{\epsilon^{2}}{2}|\Delta U|^{2} \mathrm{~d} \boldsymbol{x} .
$$

Here $\Phi$ is the strain energy potential, $\rho$ is the mass density in the reference configuration, $U=(u, v)$ is the current configuration, $\boldsymbol{F}=\nabla U$ is the deformation gradient, $t$ denotes time, $\epsilon$ is the capillarity and $\boldsymbol{x}$ is the spatial coordinate in the reference configuration. By introducing a dissipation potential $G\left(\boldsymbol{F}, \boldsymbol{F}_{t}\right)$, an equation of motion can be obtained,

$$
\rho U_{t t}=\nabla \cdot \boldsymbol{\sigma}(\boldsymbol{F})+\beta \nabla \cdot \boldsymbol{\tau}\left(\boldsymbol{F}, \boldsymbol{F}_{t}\right)-\epsilon^{2} \Delta^{2} U,
$$

\footnotetext{
a e-mail: muite@maths.ox.ac.uk
}

b e-mail: salman@onera.fr 
in which $\boldsymbol{\tau}=\nabla_{\boldsymbol{F}_{t}} G$ and $\boldsymbol{\sigma}=\nabla_{\boldsymbol{F}} \boldsymbol{\Phi}$. This framework allows comparison of geometrically nonlinear and linear models of martensitic phase transformations. Ultrasonic techniques can be used to measure the dispersion relation in a material from which it is possible to estimate elastic, dissipation and capillarity constants using the geometrically linear model $[4,12,17]^{1}$. By using a strain energy function of Saint-Venant Kirchhoff type, the same parameters can be used in a geometrically nonlinear model that is suitable for moderate strains and large rotations [19, p. 250].

The strain tensor invariants which characterize the square to rectangle transformation are $[21]$

$$
\eta_{1}=e_{11}+e_{22}, \quad \eta_{2}=e_{11}-e_{22}, \quad \eta_{3}=e_{12}=e_{21}
$$

where $e_{i j}$ is the $i j$ entry of the linear strain tensor,

$$
\boldsymbol{E}_{L}=\left(\boldsymbol{F}+\boldsymbol{F}^{T}-2 \boldsymbol{I}\right) / 2
$$

or its nonlinear counterpart,

$$
\boldsymbol{E}_{N L}=\left(\boldsymbol{F}^{T} \boldsymbol{F}-\boldsymbol{I}\right) / 2 .
$$

The second invariant corresponds to the deviatoric strain and is the order parameter which characterizes the square to rectangle transformation. Jacobs [21] simple strain energy model for the square to rectangle transformation is

$$
\Phi=a_{1}\left(\eta_{2}^{2}+a_{2}\right)\left(\eta_{2}^{2}-a_{3}\right)^{2}+b_{1} \eta_{1}^{2}+b_{2} \eta_{3}^{2} .
$$

The constants $a_{1} \geq 0, a_{2} \geq 0, a_{3}>0, b_{1} \geq 0$ and $b_{2} \geq 0$ are chosen so that $\Phi$ is bounded from below and has multiple minima at different values of $\eta_{2}$ and with $\eta_{1}=\eta_{3}=0$.

A frame indifferent dissipation potential is

$$
\begin{aligned}
G_{N L}\left(\boldsymbol{F}, \boldsymbol{F}_{t}\right) & =\beta \operatorname{det}(\boldsymbol{F})^{2} \operatorname{Tr}\left[\boldsymbol{F}_{t} \boldsymbol{F}^{-1}+\left(\boldsymbol{F}_{t} \boldsymbol{F}^{-1}\right)^{T}\right]^{2} \\
& =\beta \operatorname{Tr}\left\{\boldsymbol{F}_{t} \operatorname{adj}(\boldsymbol{F})+\left[\boldsymbol{F}_{t} \operatorname{adj}(\boldsymbol{F})\right]^{T}\right\}^{2}
\end{aligned}
$$

where $\beta$ is the viscosity. In geometrically linear viscoelasticity, the linearization of the geometrically nonlinear dissipation will be used and is given by the Rayleigh dissipation function

$$
G_{L}\left(\boldsymbol{F}, \boldsymbol{F}_{t}\right)=\beta \operatorname{Tr}\left(\boldsymbol{F}_{t}+\boldsymbol{F}_{t}^{T}\right)^{2} .
$$

\section{Numerical Results}

The evolution equations are solved using Fourier spectral collocation methods with an implicit finite difference timestepping scheme [30]. The simulations use $512 \times 512$ grid points. The simulation box is $0.1 \mu \mathrm{m} \times 0.1 \mu \mathrm{m}$, which as shown in Fig. 1 is the length scale for which microstructure is visible. To relate the model parameters to experimental values, it will be assumed that the computational sample has a depth of $0.1 \mu \mathrm{m}$. In all examples shown here, the simulations started with zero initial velocity and the low strain energy initial configuration given by

$$
\begin{gathered}
f=\tanh \{100 \sin [10(x+y+0.5) \pi] \sin [20(y-x+0.25) \pi]\}, \\
\left.u=x+10^{-4}(f-1) \sin [70(x-y) \pi]+10^{-4}(f+1) \cos [70(y+x) \pi)\right]+10^{-6} \sin (20 x \pi)
\end{gathered}
$$

\footnotetext{
${ }^{1}$ Ultrasonic studies $[4,17]$ typically do not include dissipation effects when fitting analytical expressions for the dispersion relation to experimental results. This can give a capillarity term with the wrong sign.
} 


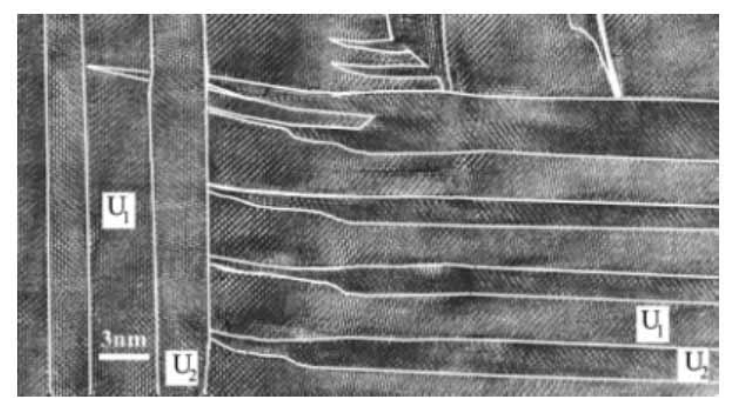

Fig. 1. A macro-twin interface in $\mathrm{NiAl}[36]$.

and

$$
v=y+10^{-4}(f-1) \sin [70(x-y) \pi]-10^{-4}(f+1) \cos [70(y+x) \pi],
$$

where $x$ and $y$ measured in $\mu m$.

The initial configuration is shown in Fig. 2(a). To compare our results to physical experiments, parameters that are reflective of the martensitic material $\mathrm{NiAl}$ are used. $\mathrm{NiAl}$ is examined because the maximum change in the entries of the martensite transformation matrix relative to the identity (the austenite phase) is approximately $13 \%$. This is larger than in most other martensitic transformations [9, p. 49-56]. Furthermore, as demonstrated in Fig. 1, microstructures in $\mathrm{NiAl}$ have bent needles and interesting macrotwin configurations $[10,36,37]$. Numerical results not presented here show that in small domains, when smaller maximum transformation strains are used, the differences in microstructural morphology between the geometrically linear and geometrically nonlinear models are not as pronounced.

A model where $\Phi$ has minima only for the martensite phases is simulated. At these minima, the symmetric and positive definite strain matrices in the geometrically nonlinear theory are $\boldsymbol{F}=[1.118,0 ; 0,0.866]$ and $\boldsymbol{F}=[0.866,0 ; 0,1.118]$. In the geometrically linear theory, $\Phi$ is minimized at $\boldsymbol{F}=[1.125,0 ; 0,0.875]$ and $\boldsymbol{F}=[0.875,0 ; 0,1.125]$. These choices correspond to $\Phi$ being minimized at $\eta_{2}= \pm 0.25$, in both the geometrically nonlinear and geometrically linear theories.Thus $a_{3}=0.0625$. No attempt is made to fit elastic constants in all directions, and for simplicity, simulations are done with $b_{1}=b_{2}=10^{11} \mathrm{Kg} / \mathrm{m} / \mathrm{s}^{2}$ to obtain elastic constants of the correct order of magnitude for martensitic systems $[2,12,26,29]$. To fix $\Phi, a_{1}=10^{11} \mathrm{Kg} / \mathrm{m} / \mathrm{s}^{2}$ and $a_{2}=0.05$ are chosen so that microstructures computed in both models resembled those observed in experiments as closely as possible. NiAl has a density of $6,600 \mathrm{Kg} / \mathrm{m}^{3}[12,29]$. The capillarity is chosen to be $\epsilon^{2}=2.5 \times 10^{-11} \mathrm{Kg} / \mathrm{m} / \mathrm{s}^{2}$ so that smooth interfaces can be resolved numerically. As explained by Dondl et al. [16], this gives a minimum interface width of approximately $6 \mathrm{~nm} \approx \sqrt{0.25 \epsilon^{2} /\left(a_{1} a_{2} a_{3}^{2}\right)}$. This interface width is up to twenty times larger than typically observed, but allows for fast computations. To test the effect of this, computational results with $\epsilon^{2}=2.5 \times 10^{-9} \mathrm{Kg} / \mathrm{m} / \mathrm{s}^{2}$ are also presented. Analytical studies suggest that the ratio $\beta^{2} / \epsilon^{2}$ is important in determining the timescale for metastable states [18,32]. A larger viscosity of $\beta=0.15 \mathrm{Kg} / \mathrm{m} / \mathrm{s}$ is used instead of the experimentally measured value of $\beta=0.015 \mathrm{Kg} / \mathrm{m} / \mathrm{s}[2,33]$, because the smallest value of $\epsilon^{2}$ is larger than expected. Since the simulations are two dimensional and it has not been possible to fit realistic strain, dissipation and capillarity potentials, only qualitative agreement between computed and experimentally observed microstructure morphology is expected.

Figure 2 shows that in both the geometrically linear and nonlinear models, there is an initial fast transient to a metastable state which then evolves slowly. Jumps in the kinetic energy which can be seen in Fig. 2 correspond to changes in microstructure topology and these are related to acoustic emission and avalanches that have been observed in other numerical studies $[1,35]$. These jumps occur more frequently and over longer time periods in the geometrically nonlinear model.

Figure 4 shows that in the geometrically linear model, once a critical time has been reached, the metastable microstructure relaxes to become a simple laminate. Similar behavior is observed 


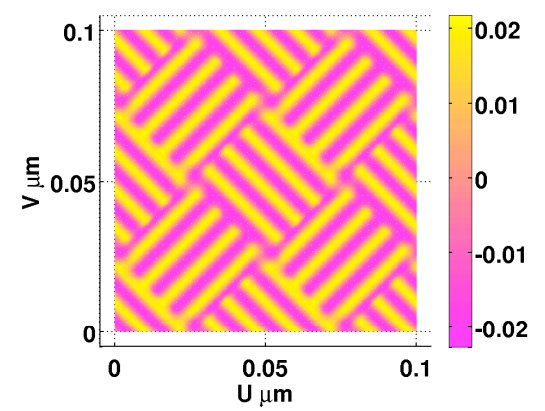

(a) Initial configuration. Colors show $\eta_{2}$ in the geometrically nonlinear model.

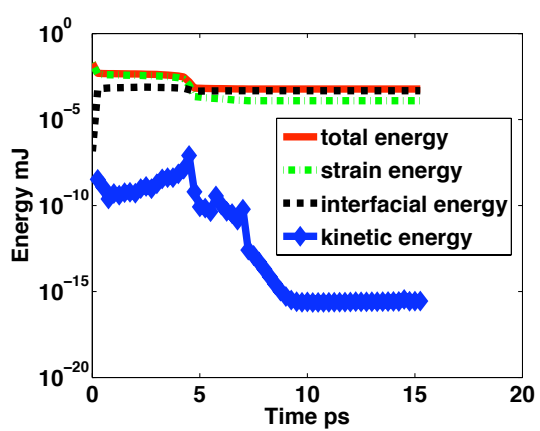

(c) Geometrically linear theory.

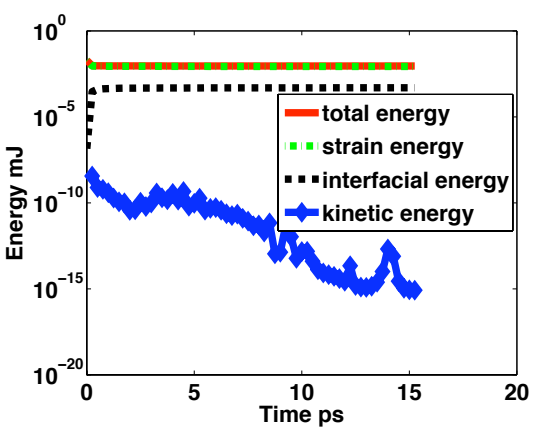

(b) Geometrically nonlinear theory.

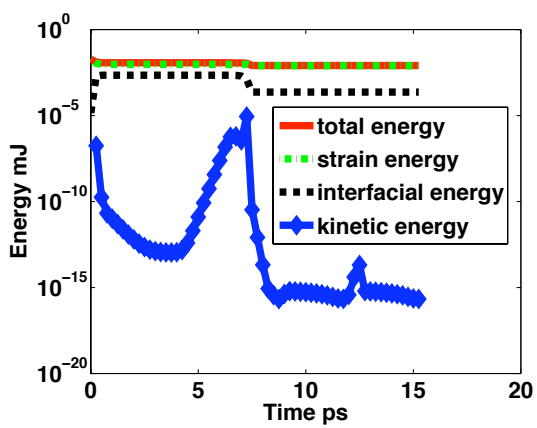

(d) Geometrically nonlinear theory larger capillarity.

Fig. 2. Energy evolution and initial iterate.

in the geometrically nonlinear model with large capillarity as shown in Fig. 5 . Figure 3 shows that the numerical solution to the geometrically nonlinear model with small capillarity evolves slowly. The morphology of the microstructure in Fig. 3(c) is close to that observed in experiment shown in Fig. 1. Figure 3(d) also shows that most of the energy is concentrated along boundaries between martensitic variants.

\section{Discussion}

There are several other computations of geometrically nonlinear models for martensitic phase transformations in the literature $[5,6,16,22,27,28]$, but none of these has compared geometrically linear and geometrically nonlinear models. Only Dondl et al. [16], Jacobs [22] and Klouček and Luskin [24] simulate geometrically nonlinear models which include a capillarity term. Neither Dondl et al. [16] nor Klouček and Luskin [24] simulate macrotwin interfaces. Jacobs [22] uses conjugate gradient energy minimisation to find low energy states. Jacobs [22] shows that tip splitting is either a low energy or long lived metastable energy state, but this study does not use very high spatial resolution or show that the persistence of the split tips is due to geometric nonlinearity which introduces many metastable states.

Kerr et al. [23] observed transient tip splitting in a geometrically linear phase field model but did not find this as a long lived metstable state. Levitas and Lee [26] observed long lived microstructures in a geometrically linear model in the presence of large defects, but experiments by Schryvers and his co-authors $[10,36,37]$ do not show large defects near split tips. The results presented here show that even without large defects, the geometrically nonlinear model gives rise to long lived microstructures that are similar to those observed in experiments. 


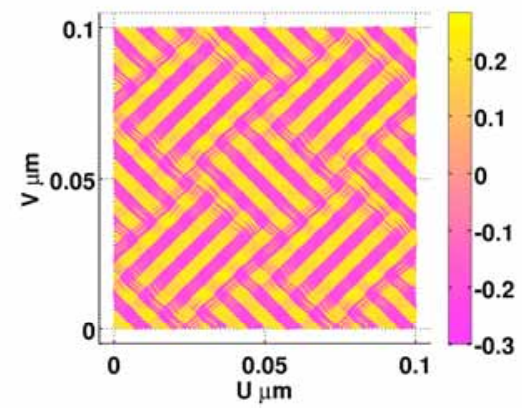

(a) 1 ps. Colors show $\eta_{2}$.

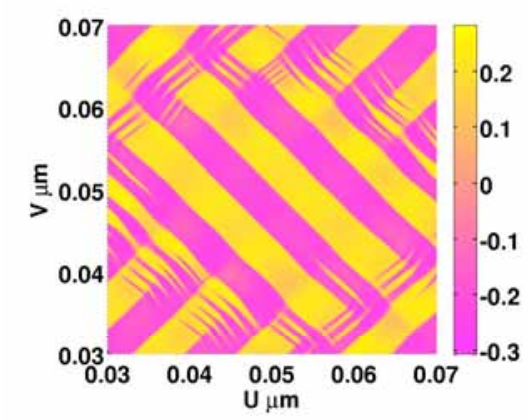

(c) 15 ps. Closeup of needles. Colors show $\eta_{2}$.

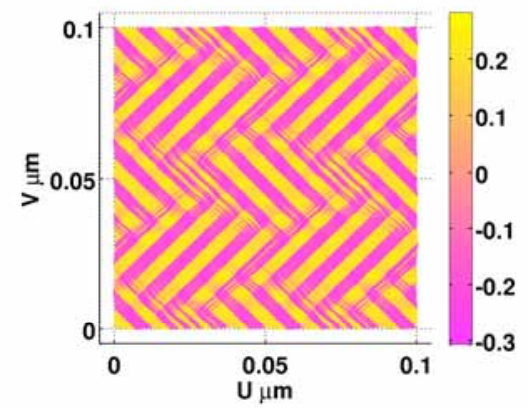

(b) 15 ps. Colors show $\eta_{2}$.

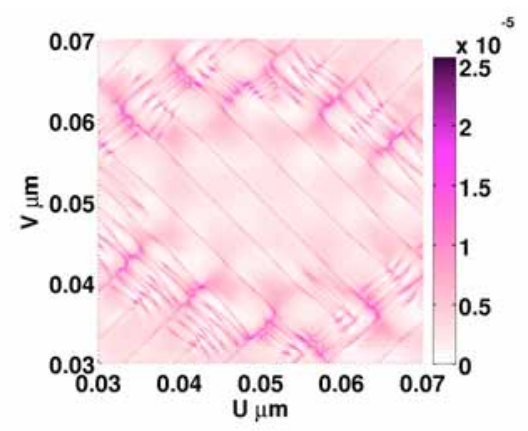

(d) 15 ps. Closeup of needles. Colors show energy density in $\mathrm{mJ} / \mu \mathrm{m}^{2}$.

Fig. 3. Deformed configurations during geometrically nonlinear evolution.

The numerical results show that the metastable states observed in the geometrically nonlinear viscoelastic models eventually relax to become simple laminates - as explained by Dolzmann and Müller $[14,15]$, this is to be expected in models with interfacial energy. A surprising result is that for the geometrically nonlinear model with small surface energy, we observe long lived metastable states for which a continuum of points on the wells participate. Such states are expected minimisers for geometrically nonlinear models without surface energy as explained by Pedregal [34, p. 59]. Our results indicate that the smaller the capillarity, the longer lived these metastable states are. Hattori and Mischaikow [18] give a proof of exponentially slow relaxation in scalar one dimensional models, and we might expect similar behavior here.

Dolzmann and Müller $[14,15]$ have also shown that minimisers for the geometrically linear and geometrically nonlinear rigid well models without interfacial energy can be very different in the presence of boundary conditions. The results presented here show that with small interfacial energy and periodic boundary conditions, long lived states for non-rigid well geometrically linear and geometrically nonlinear models also differ considerably. The numerical results support an idea expressed in Ball [7, p. 40], that study of models with small interfacial energy can also give insight into models without interfacial energy.

The duration of the metastable states is strongly influenced by geometric nonlinearity and the size of the capillarity and viscosity terms. It is also likely to depend on the height between the wells. As explained by Dondl, Shen and Bhattacharya [16] it is possible to use potentials with greater heights between the wells which, if the experimental situation can really be represented by a two-well model, should give metastable patterns that persist on physically realistic timescales of months or years instead of the microseconds calculated here. It is unclear whether such heights are physically realistic. We note that Dolzman and Müller [14] also prove that in three dimensions, only simple laminates should occur for minimisers of the three well geometrically linear cubic to tetragonal model with interfacial energy - they conjecture similar results 


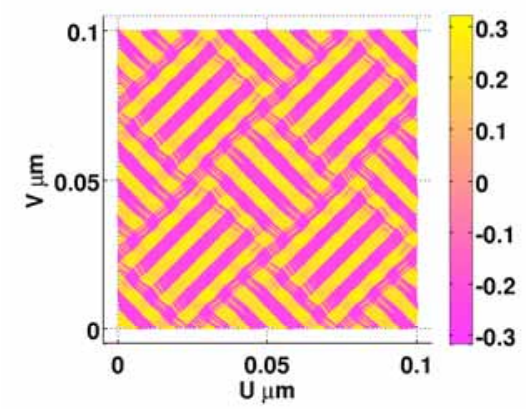

(a) 1 ps.

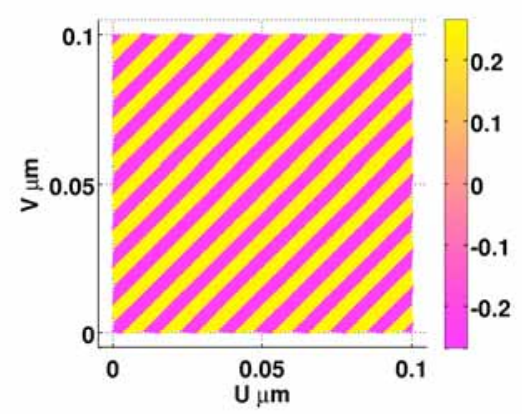

(c) 5 ps.

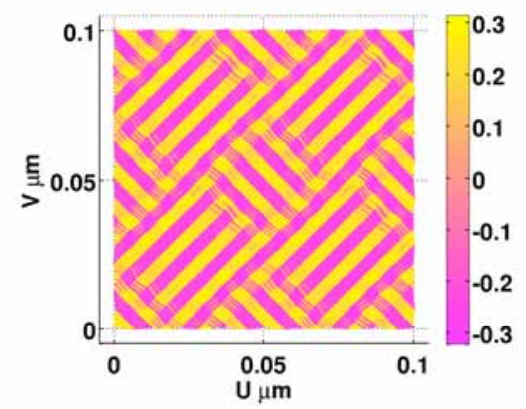

(b) 3 ps.

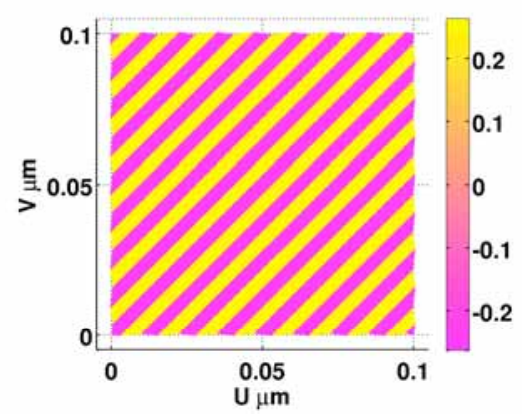

(d) $15 \mathrm{ps}$.

Fig. 4. Deformed configurations during geometrically linear evolution. Colors show $\eta_{2}$.

for the geometrically nonlinear model, but this is still unproved. Nevertheless, this suggests that these metastable states may also be relevant in the realistic three dimensional setting.

Alternative possibilities to give long lived metastable states are anisotropic dissipation and interfacial energy terms. The interfacial energy term we have included, $\frac{\epsilon^{2}}{2}|\Delta U|^{2}$, is crude and gives an interface width significantly larger than is observed in most experiments. It is also contentious as to how to interpret such a model as an approximation for atomistically sharp interfaces observed in some martensitic materials. The physics of interfaces is rather complicated, see for example, Sutton and Baluffi [38], and so the simple term used here will not capture all relevant physical effects. Ball and Mora-Corral [8] analyse a multiscale interface model and also suggest some other models which may better capture interface behavior.

It would be interesting to extend these computations to a three dimensional model for a martensitic phase transformation, for which it would be possible to perform realistic comparisons to experiments. We note that in the three dimensional case, much less is known about the possible microstructures in the case with rigid wells and no surface energy, see for example Dolzmann [13] or Müller [31]. Most of the information available consists of explicit constructions, many of which can be found in Bhattacharya [9, p. 105]. Numerical simulations here would truly be an enlightening and exploratory tool. Finally, we note that it would also be interesting to augment this viscoelastic model with a plasticity model, both to represent the motion of interfaces and to better explain energy dissipation. We leave this for future work.

\section{Acknowledgments}

We are very grateful to A. Finel for many suggestions during the course of this work. We also thank J. Ball, K. Bhattacharya, E. Crooks, and P. Plechác for helpful discussions. We acknowledge an enlightening discussion with P. Constantin on limiting processes. This work was carried 


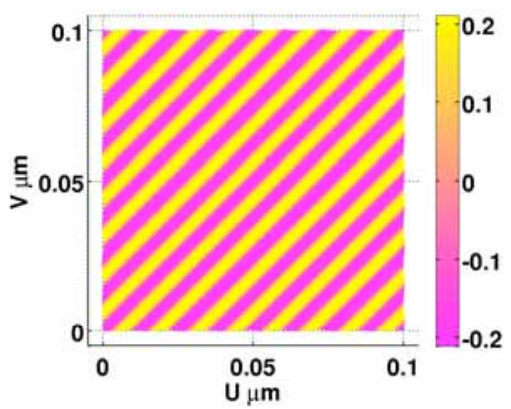

(a) 1 ps.

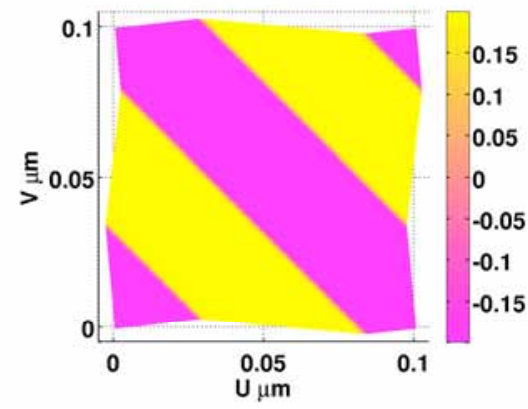

(c) 8 ps.

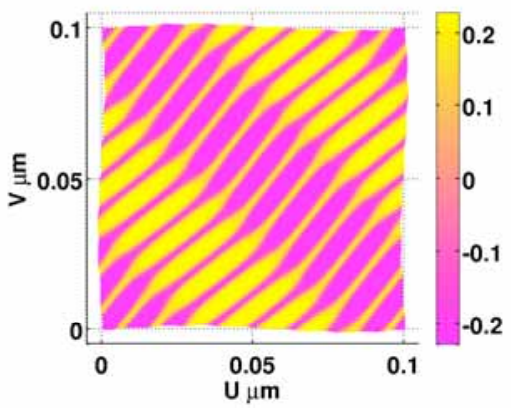

(b) 7 ps.

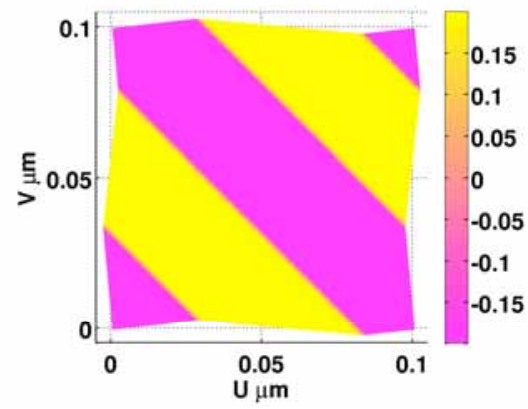

(d) $15 \mathrm{ps}$.

Fig. 5. Deformed configuration during geometrically nonlinear evolution with larger capillarity. Colors show $\eta_{2}$.

out with the support of the Marie Curie Research Training Network MULTIMAT (MRTN-CT2004-505226) and the HPC-EUROPA project (RII3-CT-2003-506079). We acknowledge computational resources provided by the Institut du developpement et des ressources en informatique scientique and the Warwick Center for Scientific Computing.

\section{References}

1. R. Ahluwalia and G. Ananthakrishna. Power-law statistics for avalanches in a martensitic transformation. Phys. Rev. Lett., 86:4076-4079, 2001.

2. R. Ahluwalia, T. Lookman, and A. Saxena. Dynamic strain loading of cubic to tetragonal martensites. Acta Materialia, 54:2109-2120, 2006.

3. S.S. Antman. Nonlinear problems of elasticity. Springer, 2005.

4. G.A. Antonelli, H.J. Maris, S.G. Malhotra, and J.M.E. Harper. Picosecond ultrasonics study of the vibrational modes of a nanostructure. J. Appl. Phys., 91(5):3261-3267, 2002.

5. M. Arndt, M. Griebel, V. Novák, P. Şittner, and T. Roubíček. Martensitic transformation in NiMnGa single crystals: Numerical simulation and experiments. Int. J. of Plasticity, 22(10):19431961, 2006.

6. M. Arndt, M. Griebel, and T. Roubíček. Modelling and numerical simulation of martensitic transformation in shape memory alloys. Cont. Mech. Thermodyn., 15:463-485, 2003.

7. J.M. Ball. Some open problems in elasticity. In Geometry, Mechanics and Dynamics, pages 3-59. Springer, 2002.

8. J.M. Ball and C. Mora-Corral. A variational model allowing both smooth and sharp phase boundaries in solids. Commmunications on Pure and Applied Analysis, 8(1):55-81, 2009.

9. K. Bhattacharya. Microstructure of martensite: why it forms and how it gives rise to the shapememory effect. Oxford University Press, 2003. 
10. P. Boullay, D. Schryvers, and R.V. Kohn. Bending martensite needles in $\mathrm{Ni}_{65} \mathrm{Al}_{35}$ investigated by two-dimensional elasticity and high-resolution transmission electron microscopy. Phys. Rev. B, 64:144105, 2001.

11. M. Bouville and R. Ahluwalia. Effect of lattice-mismatch-induced strains on coupled diffusive and displacive phase transformations. Phys. Rev. B, 75:054110, 2007.

12. T. Davenport, L. Zhou, and J. Trivisonno. Ultrasonic and atomic force studies of the martensitic transformation induced by temperature and uniaxial stress in nial alloys. Phys. Rev. B, 59(5):34213426, 1999.

13. G. Dolzmann. Variational methods for Crystalline Microstructure - Analysis and Computation. Springer-Verlag, 2003.

14. G. Dolzmann and S. Müller. The influence of surface energy on stress-free microstructures in shape memory alloys. Meccanica, 30:527-539, 1995.

15. G. Dolzmann and S. Müller. Microstructures with finite surface energy: the two-well problem. Arch. Rational Mech. Anal., 132:101-141, 1995.

16. P. Dondl, C.-P. Shen, and K. Bhattacharya. Computational analysis of martensitic thin films using subdivision surfaces. Int. J. Numer. Meth. Engng., 72:72-94, 2007.

17. H.Y. Hao and H.J. Maris. Experiments with acoustic solitons in crystalline solids. Phys. Rev. B, 64:064302, 2001.

18. H. Hattori and K. Mischaikow. On the slow motions of phase boundaries in the Korteweg theory of capillarity. Dynamic Systems and Applications, 1:441-452, 1992.

19. G.A. Holzapfel. Nonlinear solid mechanics. Wiley, 2000.

20. P. Howell, G. Kozyreff, and J. Ockendon. Applied Solid Mechanics. Cambridge University Press, 2009.

21. A.E. Jacobs. Solitons of the square-rectangular martensitic transformation. Phys. Rev. B, 31(9):5984-5989, 1985.

22. A.E. Jacobs. Landau theory of structures in tetragonal-orthorhombic ferroelastics. Phys. Rev. B., 61(10):6587-6595, 2000.

23. W.C. Kerr, M.G. Killough, A. Saxena, P.J. Swart, and A.R. Bishop. Role of elastic compatibility in martensitic texture evolution. Phase Transitions, 69(3):247-270, 1999.

24. P. Klouček and M. Luskin. Computational modeling of the martensitic transformation with surface energy. Mathl. Comput. Modelling, 20(10/11):101-121, 1994.

25. L.D. Landau and E.M. Lifshitz. Theory of elasticity. Course of theoretical physics. ButterworthHeinemann, 1984.

26. V.I. Levitas and D.-W. Lee. Athermal resistance motion in the phase-field theory of microstructure evolution. Phys. Rev. Lett., 99:245701, 2007.

27. B. Li and M. Luskin. Theory and computation for the microstructure near the interface between twinned layers and a pure variant of martensite. Mat. Sci. Eng. A, 273-275:237-240, 1999.

28. M. Luskin. On the computation of crystalline microstructure. Acta Numerica, 5:191-258, 1996.

29. Y. Mishin, M.J. Mehl, and D.A. Papaconstantopoulos. Embedded-atom potential for B2-NiAl. Phys. Rev. B, 65:223114, 2002.

30. B.K. Muite. Analysis, modeling and simulation of shape memory alloys. D. Phil thesis, University of Oxford, in preparation, 2009.

31. S. Müller. Calculus of Variations and geometric evolution problems, volume 1713 of Lecture Notes in Math., chapter Variational models for microstructure and phase transitions, pages $85-210$. SpringerVerlag, 1999.

32. B. Nicolaenko and W. Qian. Inertial manifolds for nonlinear viscoelasticity equations. Nonlinearity, 11:1075-1093, 1998.

33. M.M. Parlinska-Wojtan. Martensitic phase transformation of NiTi-based shape memory alloys: characterization and application. PhD thesis, École Polytechnique Fédérale de Lausanne, 2002.

34. P. Pedregal. Variational Methods in Nonlinear Elasticity. SIAM, 2000.

35. O.U. Salman. Modeling of spatio-temporal dynamics and patterning mechanisms of martensites by phase-field and Lagrangian methods. PhD thesis, Docteur de L'Universite de Pierre et Marie Curie, 2009.

36. D. Schryvers, P. Boullay, R.V. Kohn, and J.M. Ball. Lattice deformations at martensite-martensite interfaces in Ni-Al. J. de Physique IV, 11:23-30, 2001.

37. D. Schryvers, P. Boullay, P.L. Potapov, R.V. Kohn, and J.M. Ball. Microstructures and interfaces in Ni-Al martensite: comparing HRTEM observations with continuum theories. Int. J. Solids and Struct., 39:3543-3554, 2002.

38. A.P. Sutton and R.W. Baluffi. Interfaces in crystalline materials, volume 51 of Monographs on the physics and chemistry of materials. Clarendon Press, 1995. 